\title{
New observational results on pulsating B stars
}

\author{
A. Pigulski \\ Wrocław University Observatory, Kopernika 11, 51-622 Wrocław, Poland
}

\begin{abstract}
The paper summarizes recent observational results concerning pulsating main-sequence Btype stars: $\beta$ Cephei and SPB. In particular, recent studies of Galactic open clusters and Magellanic Clouds led to the discovery of many $\beta$ Cephei stars. These results confirm observationally the strong dependence of the pulsation mechanism of $\beta$ Cephei stars on metallicity, predicted by theory. New findings in the follow-up observations of SPB stars and asteroseismology of $\beta$ Cephei stars are also described.
\end{abstract}

\section{Introduction}

Theory and observations support each other in bringing new ideas which lead to a better understanding of stars and their evolution. The pulsations of main-sequence B-type pulsators, $\beta$ Cephei and SPB, were explained successfully a decade ago. The success of theory enabled some detailed predictions which could be now tested observationally. In particular, the effect of metallicity on pulsations can be checked in a great detail. In this context, there are actually two main trends in the observational efforts concerning the two types of variable stars mentioned above: (i) search for new variable stars, (ii) detailed studies of selected stars. This short paper summarizes recent observational results in these areas.

\section{Galactic $\beta$ Cephei stars}

In 1993, Sterken \& Jerzykiewicz published a list of 59 certain $\beta$ Cephei stars supplemented by a list of 79 suspects. Out of the former list, 17 stars were members of three southern clusters (NGC 3293, NGC 4755, and NGC 6231) that in the past helped to establish that $\beta$ Cephei stars are main-sequence objects (Balona \& Engelbrecht 1981, Balona \& Shobbrook 1983). The remaining stars from the list could be assigned either to Galactic OB associations or the Galactic field. At that time not a single certain $\beta$ Cephei-type star was known in an open cluster of the northern hemisphere. Since the publication of that paper, twelve new $\beta$ Cephei stars were found in the three famous southern clusters (Koen 1993; Balona \& Koen 1994; Balona 1994; Arentoft et al. 2001; Stankov et al. 2002) bringing the total number of their member $\beta$ Cephei stars to 29 , roughly one third of all Galactic stars of this type.

During the last decade, about $15 \beta$ Cephei stars were also discovered in the northern open clusters. It was mainly the result of the observing programs undertaken in the Wroctaw University Observatory and other Polish observatories. Among others, $\beta$ Cephei stars were discovered in h \& $\chi$ Persei (Krzesiński \& Pigulski 1997, Krzesiński et al. 1999, GomezForrellad 2000), NGC 663 (Pietrzyński 1997; Pigulski et al. 2001), NGC 7235 (Pigulski et al. 1997), NGC 7419 (Kołaczkowski et al. 2002), Cygnus OB2 (Pigulski \& Kołaczkowski 1998), and recently, in NGC 6910 (Kołaczkowski et al. 2004b). In addition, six stars were 
found by Waelkens et al. (1998) and Aerts (2000) in the Hipparcos photometry. Adding a few other found/confirmed in the Galactic field (Telting \& Schrijvers 1998; Robb et al. 2000, Paardekooper et al. 2002), we actually know over $90 \beta$ Cephei stars in the Galaxy.

Having searched some northern clusters for the presence of early-type variables, we can make some comparisons. In particular, the number of $\beta$ Cephei stars with respect to the number of all B-type stars in a certain range of absolute magnitude can be calculated. This study (Pigulski et al. 2002) revealed a striking difference in the incidence of this type of variability that cannot be explained as a result of differences in age or the detection threshold. Namely, while in the three southern clusters located along the Sagittarius-Carina spiral arm, $35 \pm 7 \%$ stars with $-4.5<M_{\mathrm{V}}<-1.5$ are $\beta$ Cephei pulsators, in 13 clusters of the Local and Perseus arms this number amounts to $6 \pm 2 \%$ (see Fig. 1). Since the presence of an overall metallicity gradient in the Galaxy seems to be well established (see, e.g., Chen et al. 2003), this result can be explained as observational evidence for the strong dependence of the pulsation mechanism of $\beta$ Cephei stars on metallicity.

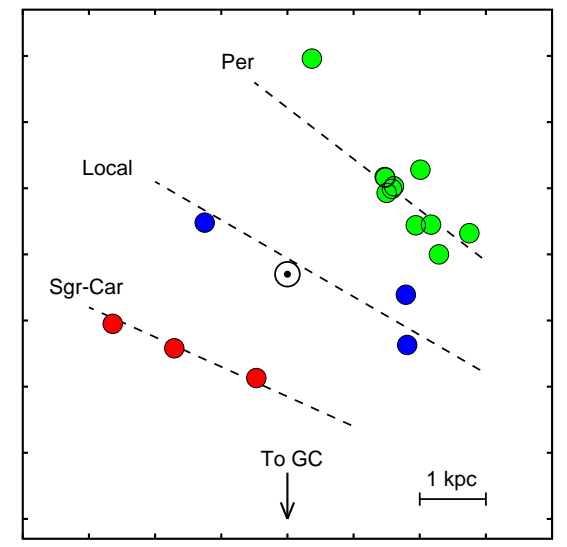

Figure 1: Location of young open clusters searched for the presence of $\beta$ Cephei stars in the Galactic plane. Direction to the Galactic center is shown by the arrow. Three nearby spiral arms are labelled. The Sun $(\odot)$ is in the center of the figure.

\section{$\beta$ Cephei stars in the LMC}

In view of the strong dependence of the pulsations of $\beta$ Cephei and SPB stars on metallicity, it is interesting to answer the question whether these variables can be found in objects of low metallicity. From the theoretical point of view (Pamyatnykh 1999), $\beta$ Cephei-type pulsations practically cease at $Z=0.01$. With the average $Z \simeq 0.008$ in the Large $(L M C)$ and $Z \simeq$ 0.004 in the Small Magellanic Cloud (SMC), pulsations should not be observed. In fact, first attempts failed to find $\beta$ Cephei stars in the Clouds. However, thanks to the microlensing surveys, huge photometric databases, OGLE-II and MACHO, are now available for the Clouds. The analysis of short-period variables from the OGLE-II catalogue of Żebruń et al. (2001) supplemented by MACHO data, allowed us to find the first three $\beta$ Cephei stars in the LMC (Pigulski \& Kołaczkowski 2002). After reprocessing the OGLE-II data, this work has been repeated. As a result of the analysis of the photometry of over 75000 early B-type stars, 64 short-period, presumably $\beta$ Cephei-type stars, were found in the LMC. This discovery nearly doubles the number of known $\beta$ Cephei stars. A preliminary report of this work has been 
recently published by Kołaczkowski et al. (2004a). In the colour-magnitude diagram of the LMC, the stars occupy mainly the range $16<V<17.5$, with only a few brighter stars (Fig. 2).

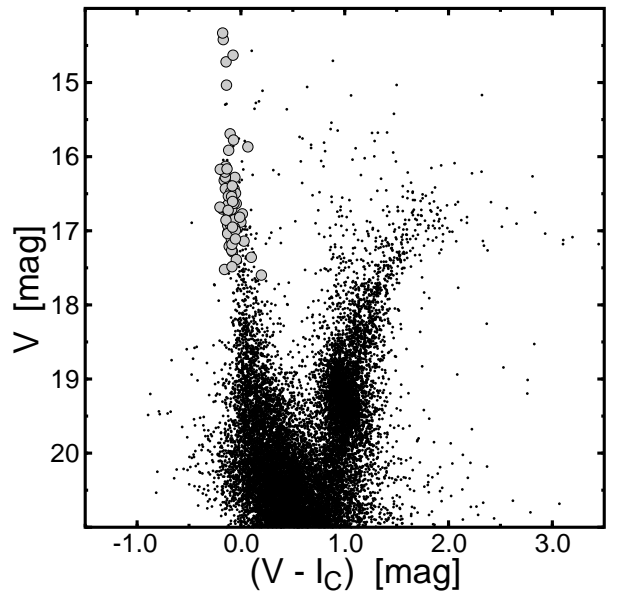

Figure 2: The colour-magnitude diagram of one of the OGLE-II LMC fields showing the positions of 64 $\beta$ Cephei stars (grey dots) found in the LMC. [Adopted from Kołaczkowski et al. (2004a)]
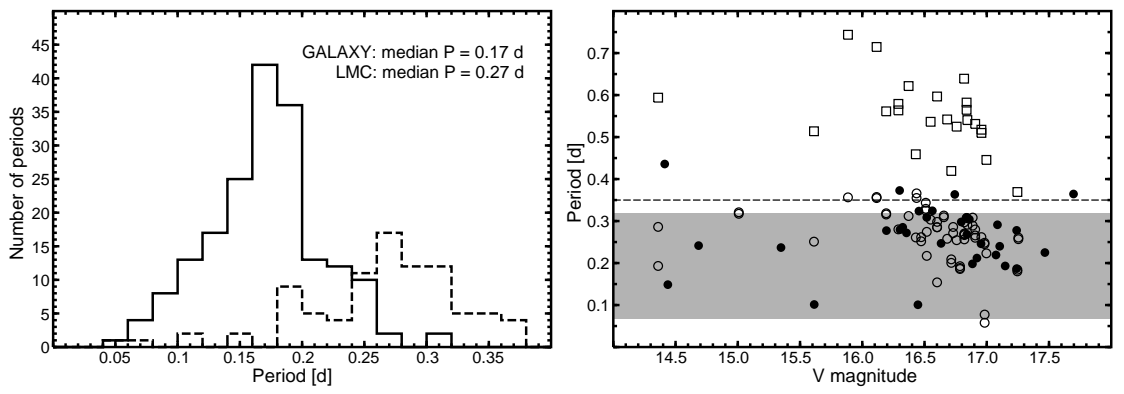

Figure 3: Left: The distribution of periods in the Galactic (solid line) and the LMC (dashed line) $\beta$ Cephei stars. Right: Periods detected in the LMC $\beta$ Cephei stars plotted against the $V$ magnitude. Periods in monoperiodic stars are shown as filled circles, those found in multiperiodic stars, as open circles (short periods) and open squares (long periods). The grey strip covers the range of periods observed in Galactic $\beta$ Cephei stars. [Adopted from Kołaczkowski et al. (2004a)]

The $\beta$ Cephei stars found in the LMC differ from the Galactic ones. First, their periods are generally longer: while the median period for Galactic stars is $0.17 \mathrm{~d}$, it reaches $0.27 \mathrm{~d}$ for the LMC objects (Fig. 3). At least partly, this can be explained by lower metallicity, because at lower metallicities the instability strip becomes narrower and confined to regions very close to the end of the hydrogen-core burning phase of evolution (Pamyatnykh 1999). However, even more exciting is the fact that out of $64 \beta$ Cephei stars in the LMC, twenty $(\sim 30 \%)$ 
show additional period(s) longer than $\sim 0.35 \mathrm{~d}$ (Fig. 3). So far, such long periods were not observed in the Galactic $\beta$ Cephei stars. These periods need to be explained theoretically; they can be probably attributed to low-order $g$ modes.

\section{Asteroseismology of $\beta$ Cephei stars}

Asteroseismology of $\beta$ Cephei stars is still in its infancy. Using this promising tool we would like to investigate the internal chemical and rotation profiles, presence of magnetic field, core overshooting, etc. In order to get this information, we first need to detect modes and then identify them properly. The first attempts have been already made for some well-studied $\beta$ Cephei stars: 16 (EN) Lac (Dziembowski \& Jerzykiewicz 1996), $\beta$ Cep (Shibahashi \& Aerts 1998), 12 (DD) Lac (Dziembowski \& Jerzykiewicz 1999), V836 Cen (Aerts et al. 2003), IL Vel, V433 Car, and KZ Mus (Handler et al. 2003), and finally, $\nu$ Eri. The latter star was observed within a large international campaign (Handler \& Aerts 2003a,b) and actually has the largest number of independent modes detected: nine.

As yet, the asteroseismology of $\beta$ Cephei stars did not teach us much about these stars. Simply the number of modes is too small, too few rotationally split multiplets are observed, we still lack reliable mode identifications. It also happens that the models we use are too simple or inadequate. The models help in many cases to reject some identification and constrain global stellar parameters, but we are still far from the real asteroseismological work. There is probably only an indication for faster core rotation coming from some papers mentioned above (Dziembowski \& Jerzykiewicz 1996, Aerts et al. 2003).

\section{New photometry and spectroscopy of SPB stars}

About a dozen of slowly pulsating B stars (SPB, Waelkens 1991), showing high-order $g$ modes, were known before the Hipparcos mission. Hipparcos increased this number to about a hundred (Waelkens et al. 1998). It soon became clear, however, that with Hipparcos photometry alone, only a limited number of modes can be detected. Moreover, misidentifications are quite possible, so that follow-up observations are highly desirable. This is also because SPB stars are not easy to observe. The periods of the order of 1 day, small amplitudes and a dense spectrum of excited modes, make the mode detection a challenging task. Also, some other kinds of variability are indistinguishable in photometry from single-mode SPB-type pulsations. With about a hundred data points from Hipparcos distributed unevenly over three years, the study of multiperiodicity becomes very difficult (see, e.g., Molenda-Żakowicz 2002). For this reason, Aerts et al. (1999) initiated a program of follow-up spectroscopic and photometric observations of brightest southern SPB stars found by Hipparcos, extended later to the Northern hemisphere (Mathias et al. 2001). As a result, many spectroscopic binaries were found (De Cat et al. 2000) and some stars were reclassified as ellipsoidal or chemically peculiar stars (De Cat \& Aerts 2002). At present, about 40 SPB stars have a secure classification (De Cat 2003).

SPB stars were also detected in the photometric studies of open clusters. For example, they were found in NGC 7654 (Choi et al. 1999), h Per (Krzesiński et al. 1999), NGC 3293 (Arentoft et al. 2001), and NGC 4755 (Stankov et al. 2002). At present, about 20 SPB stars are known in Galactic clusters.

Finally, SPB stars were discovered in the LMC. First examples were reported by Kołaczkowski et al. (2003), but a comprehensive study that is now in progress will surely bring a bulk of new discoveries. For the study of these stars, the microlensing databases, covering presently about 10 years and having no large gaps, constitute an ideal source of photometry. 


\section{Final comments}

Undoubtedly, there are great prospects in studying $\beta$ Cephei and SPB stars in Galactic clusters and nearby galaxies, especially in the Magellanic Clouds. The studies of this kind will help to understand the role of metallicity in the pulsations and will allow us to find the observational limits of metallicity required to get driving. It is obvious that spectroscopic determinations of abundances will be needed to establish this quantitatively. In an even broader context, some difficult and important questions on the stability and mode selection could be answered. On the other hand, photometric campaigns on selected objects are highly desirable as they, by means of the asteroseismological methods, would at least allow some constraints to be put on global stellar parameters and mode identification of these variable stars.

Acknowledgments. This paper has been supported by the KBN grant 5 P03D 01420 .

\section{References}

Aerts, C. 2000, A\&A 361, 245

Aerts, C., De Cat, P., Peeters, E. et al. 1999, A\&A 343, 872

Aerts, C., Thoul, A., Daszyńska, J. et al. 2003, Science 300, 1926

Arentoft, T., Sterken, C., Knudsen, M.R. et al. 2001, A\&A 380, 599

Balona, L.A. 1994, MNRAS 268, 119

Balona, L.A., Engelbrecht, C. 1981, Workshop on pulsating B stars, p. 195, eds. G.E.V.O.N. \& C.Sterken Balona, L.A., Koen, C. 1994, MNRAS 267, 1071

Balona, L.A., Shobbrook, R.R. 1983, MNRAS 205, 309

Chen, L., Hou, J.L., Wang, J.J. 2003, A\&A 401, 939

Choi, H.S., Kim, S.-L., Kang, Y.H., Park, B.-G. 1999, A\&A 348, 789

De Cat, P. 2003, Proc. IAU Coll. 193, in press

De Cat, P., Aerts, C. 2002, A\&A 393, 965

De Cat, P., Aerts, C., De Ridder, J. 2000, A\&A 355, 1015

Dziembowski, W., Jerzykiewicz, M. 1996, A\&A 306, 436

Dziembowski, W., Jerzykiewicz, M. 1999, A\&A 341, 480

Gomez-Forrellad, J.M. 2000, IBVS No. 4924

Handler, G., Aerts, C. 2003a, Comm. in Asteroseismology 142, 20

Handler, G., Aerts, C. 2003b, Proc. IAU Coll. 193, in press

Handler, G., Shobbrook, R.R., Vuthela, F.F. et al. 2003, MNRAS 341, 1005

Koen, C. 1993, MNRAS 264, 165

Kołaczkowski, Z., Pigulski, A., Kopacki, G. 2002, A.S.P. Conf. Ser. 259, 150

Kołaczkowski, Z., Pigulski, A., Soszyński, I. et al. 2004a, ASP Conf. Ser., in press (astro-ph/0310582)

Kołaczkowski, Z., Kopacki, G., Pigulski, A., Michalska, G., 2004b, Acta Astron., submitted

Krzesiński, J., Pigulski, A. 1997, A\&A 325, 987

Krzesiński, J., Pigulski, A., Kołaczkowski, Z. 1999, A\&A 345, 505

Mathias, P., Aerts, C., Briquet, M. et al. 2001, A\&A 379, 905

Molenda-Żakowicz, J. 2002, Ph.D. thesis, University of Wroctaw

Paardekooper, S.J., Veen, P.M., van Genderen, A.M., van der Hucht, K.A. 2002, A\&A 384, 1012

Pamyatnykh, A.A. 1999, AcA 49, 119

Pietrzyński, G. 1997, AcA 47, 211

Pigulski, A., Kołaczkowski, Z. 1998, MNRAS 298, 753

Pigulski, A., Kołaczkowski, Z. 2002, A\&A 388, 88

Pigulski, A., Jerzykiewicz, M., Kopacki, G. 1997, AcA 47, 365

Pigulski, A., Kopacki, G., Kołaczkowski, Z. 2001, AcA 51, 159

Pigulski, A., Kopacki, G., Kołaczkowski, Z., Jerzykiewicz, M. 2002, A.S.P. Conf. Ser. 259, 146

Robb, R.M., Delaney, P.A., Cardinal, R.D. et al., 2000, IBVS No. 4913

Shibahashi, H., Aerts, C. 2000, ApJ 531, L143

Stankov, A., Handler, G., Hempel, M., Mittermayer, P. 2002, MNRAS 336, 189

Sterken, C., Jerzykiewicz, M. 1993, Space Science Rev. 62, 95

Telting, J.H., Schrijvers, C. 1998, A\&A 339, 150

Waelkens, C. 1991, A\&A 246, 453

Waelkens, C., Aerts, C., Kestens, E., Grenon, M., Eyer, L. 1998, A\&A 330, 215

Żebruń, K., Soszyński, I., Woźniak, P.R. et al. 2001, AcA 51, 317. 